\title{
¿TE ANIMAS A LEER? UNA EXPERIENCIA DE ANIMACIÓN A LA LECTURA EN EDUCACIÓN PRIMARIA
}

\author{
Recepción: 01/11/2017 | Revisión: 29/11/2017 | Aceptación: 19/12/2017
}

\author{
Vanessa ORTEGA-QUEVEDO \\ Noelia SANTAMARÍA-CÁRDABA \\ Universidad de Valladollid \\ Universidad de Valladollid \\ vanessaortegaquevedo@gmail.com \\ noelia.santamaria.cardaba@uva.es

\section{Irene MARTÍN HIDALGO} \\ Universidad de Valladollid \\ irenemh_25@hotmail.com

\section{Félix E. LOBO DE DIEGO} \\ Universidad de Valladollid \\ flobodediego@gmail.com
}

Rosa ORTIZ DE SANTOS

Universidad de Valladollid

rosa.ortiz@uva.es

Resumen: El fin principal de esta propuesta didáctica es dar a conocer una forma de educar en el gusto por la lectura, conocida como animación a la lectura. En concreto, se emplea el método de Montserrat Sarto (Sarto, 1984, 1998), basado en tres objetivos: acercar a los jóvenes a lecturas que puedan comprender, procurar que los estudiantes disfruten con la lectura y fomentar la reflexión sobre lo leído. En esta línea, se elabora una programación de animación a la lectura dirigida al alumnado de sexto curso de Educación Primaria de un centro educativo de Segovia. Dicha propuesta se elabora a partir de las estrategias extraídas de publicaciones de referencia (Sarto, 1984, 1998). Como resultado, los participantes de esta experiencia puntual, además de mostrar interés por la lectura, han manifestado ganas por mejorar su comprensión lectora y por continuar aprendiendo con este método. En conclusión, se ha logrado cumplir el fin principal, además de los tres objetivos que propone el método de animación empleado, lo cual pone de manifiesto que la propuesta atrae la atención de los jóvenes, promoviendo un acercamiento positivo a la lectura.

Palabras clave: animación a la lectura; didáctica de la lengua; educación lectora; Educación Primaria; método de Montserrat Sarto.

\section{DO YOU FANCY READING? A MOTIVATION-TO-READ EXPERIENCE IN PRIMARY EDUCATION}

\begin{abstract}
The main aim of this didactic proposal is to show a method to educate reading comprehension among children, also known as motivation to reading. This is achieved using the Montserrat Sarto's method (Sarto, 1984, 1998), which established three objectives: approach children to readings that they can understand, promote reading enjoyment, and encourage self-reflection about what they have read. In this line, we prepared a motivation to reading experience for the sixth course of Primary Education in a state-funded school from Segovia. The reading motivation was implemented from strategies extracted from the method previously mentioned (Sarto, 1984, 1998). As a result, we observed that the participants of this specific experience showed more interest in improving their reading comprehension and wanted to continue learning with the method mentioned. In conclusion, the main aim was achieved in addition to the three objectives proposed by the animation method used, which shows that the proposal has a positive influence in grabbing the students' attention, thus promoting a positive approach to reading.
\end{abstract}

Keywords: motivation to reading; language didactics; reading education; Primary Education; Montserrat Sarto's method.

\section{T'ANIMES A LLEGIR? UNA EXPERIÈNCIA D'ANIMACIÓ} A LA LECTURA EN EDUCACIÓ PRIMÀRIA

Resum: El fi principal d'aquesta proposta didàctica és promoure una forma d'educar en el gust per la lectura, coneguda com animació a la lectura. Comcretament, s'utilitza el mètode de Montserrat Sarto (Sarto, 1984, 1998), basat en tres objectius: apropar els joves a lectures que puguin comprendre, procurar que els estudiants gaudeixin amb la lectura i fomentar la reflexió sobre el que s'ha llegit. En aquesta línia, s'elabora una programació d’animació a la lectura dirigida a l'alumnat de sisè curs d'Educació Primària d'un centre educatiu de Segòvia. Aquesta proposta s'elabora a partir de les estratègies extretes de publicacions de referència (Sarto, 1984, 1998). Com a resultat, els participants d'aquesta experiència puntual, a més de mostrar interès per la lectura, han manifestat ganes per a millorar la seva comprensió lectora i per continuar aprenent amb aquest mètode. En conclusió, s'ha aconseguit el fi principal, a més dels tres objectius que proposa el mètode d'animació utilitzat, cosa que posa de manifest que la proposta atrau l'atenció dels joves, promovent un apropament positiu a la lectura.

Paraules clau: animació a la lectura; didàctica de la llengua; educació lectora; Educació Primària; mètode de Montserrat Sarto. 


\section{Introducción}

Los resultados extraídos durante los últimos años del informe del Programa para la Evaluación Internacional PISA demuestran que el alumnado español manifiesta carencias en comprensión lectora al acabar la etapa de educación obligatoria (OCDE, 2015). En el proceso lector, los jóvenes españoles no adquieren la destreza necesaria para comprender de forma fluida textos y no suelen poner en práctica sus habilidades lectoras, poniendo así de manifiesto la tendencia de rechazo hacia la lectura. En esta línea, Artola, Sastre y Barraca (2016) esclarecen que "Los estudios realizados en la última década sugieren que las actitudes de los alumnos hacia la lectura han empeorado" (p. 13). Los estudiantes entran en un círculo que les atrapa: no leen porque no comprenden y no desarrollan su comprensión porque no ponen en práctica sus habilidades ni las mejoran. "El niño que vive de espaldas a la lectura [...] ha llegado a la conclusión de que la lectura es aburrida, incómoda y requiere un esfuerzo que él no está dispuesto a emprender" (Sarto, 1984:17).

En este sentido, se considera que "cuanto mayores sean los conocimientos previos de que disponga el lector, mayor será su capacidad para predecir y elaborar inferencias durante la lectura, así como para construir modelos adecuados del significado del texto" (Gutiérrez, 2016:53). Por ello, desde la didáctica de la lengua y la literatura se procura incentivar metodologías y propuestas didácticas que ayuden a los discentes a mejorar su comprensión lectora y a conseguir hábito lector.

Ahora bien, el hábito lector no está relacionado con la didáctica, sino con el gusto por la lectura. No se puede obligar a un estudiante a que se emocione con ella, pero sí se puede procurar educarle en el gusto por leer y, a través de la didáctica, ayudarle a desarrollar las habilidades necesarias para realizar dicha tarea (Sarto, 1998). Desde la didáctica se intenta proporcionar al alumnado los apoyos necesarios para aprender a decodificar y comprender el lenguaje escrito. Sin embargo, para mejorar el gusto lector hay que tener en cuenta otros factores disposicionales que deben tratarse de forma específica, como por ejemplo la motivación. En palabras de Gambrell (2011:172) “...si los estudiantes no están motivados para leer, nunca alcanzarán su potencial de alfabetización completo”.

En esta línea, Avendaño (2017) recoge distintos cuerpos teóricos que ofrecen perspectivas de cómo se relaciona la motivación con la lectura (motivación intrínseca y extrínseca; autoeficacia; teoría expectativa/valor); no obstante, la visión que este artículo quiere abordar es holística. Se pretende fomentar la motivación intrínseca del alumnado, es decir, su motivación de leer para disfrutar (Guthrie y Wigfield, 2000). De este modo, al alcanzar el disfrute, se promoverá que los educandos afronten la lectura con mejores expectativas y tengan en mayor consideración su autoeficacia lectora. Asimismo, es importante inculcar al alumnado el valor de la lectura, el cual incluye factores como la importancia, el interés y la utilidad de la misma (Wigfield y Eccles, 2000). Respecto a la relación existente entre estos procedimientos motivacionales y la mejora de la habilidad lectora del alumnado, conviene destacar que diversos estudios de investigación (Becker, McElvany y Kortenbruck, 2010; McGeown, Norgate y Warhurst, 2012; McGeown, Duncan y Griffiths, 2015) demuestran la veracidad de dicha relación, y por ende, la importancia de su implementación en las aulas. Con este fin se propone emplear la metodología para la educación literaria conocida como animación a la lectura.

La animación a la lectura, según Carmen Olivares, antigua directora de la librería Talen- 
tum (Madrid), es "un acto consciente realizado para producir un acercamiento afectivo e intelectual a un libro concreto, de forma que ese contacto produzca una estimación genérica hacia los libros" (Sarto, 1984:18). Es decir, la animación a la lectura no es un acto obligatorio donde se exige al educando leer, porque el acercamiento al libro sería algo negativo; no es un objetivo instrumental, pues no debe estar al servicio de otras disciplinas, y no es una forma de calificar la habilidad lectora de los discentes (Sarto, 1998).

En base a lo expuesto, este artículo presenta una experiencia didáctica basada en la metodología propuesta por Montserrat Sarto en su libro Animación a la lectura con nuevas estrategias. La autora presenta distintas estrategias basadas en el juego creativo para promover un acercamiento positivo del alumnado a una obra literaria. En consecuencia, se entiende por estrategias para desarrollar una sesión de animación a la lectura aquellos procesos utilizados para la educación en la lectura de los niños. Los objetivos perseguidos con dichas estrategias se corresponden con la inmersión del estudiante en la literatura a través de lecturas adecuadas a su nivel de comprensión lectora, promover el desarrollo de su habilidad lectora, educar el gusto por la lectura y favorecer la reflexión (Sarto, 1998). Para desarrollar una sesión de animación a la lectura que cumpla estos objetivos es necesario tener presente una serie de aspectos y elaborar cuidadosamente una programación que será ejecutada por un mediador. En este sentido, el método propuesto por Sarto $(1984,1998)$ establece que:

(1) Se debe proponer una selección de libros adaptados a la edad y la habilidad lectora del grupo al que van a ir dirigidas las sesiones. (2) Las estrategias deben escogerse con anterioridad y adaptarse al grupo escolar. Al realizar la selección, es preciso organizar la temporalización de la sesión. Asimismo, debe procurarse que las estrategias no se repitan frecuentemente entre sesiones. (3) De la selección previa de libros es preciso elegir el más adecuado para trabajar las estrategias propuestas. (4) Los discentes deben realizar una lectura individual previa no obligatoria del libro en sus hogares. Durante este proceso de trabajo individual la lectura debe ser silenciosa, pues solo así el alumnado podrá interiorizar lo que lee y, a partir de dicha interiorización, prepararse para la posterior reflexión sobre la lectura. (5) En la puesta en práctica de la sesión de animación, esta puede sufrir las adaptaciones que el animador considere precisas para que se desarrolle de forma óptima.

Cabe señalar que la no obligatoriedad de la lectura silenciosa es primordial para que el acercamiento a la obra sea positivo. Es frecuente diseñar propuestas contradictorias que pretendan crear el gusto y la pasión a partir de ejercicios forzosos, lo cual no es certero (Mata, 2008).

\section{Fundamentación metodológica}

La metodología empleada en esta experiencia didáctica se basa en los trabajos de Montserrat Sarto para llevar a cabo el acercamiento de los jóvenes a la lectura, a través de la animación. La autora transmite la necesidad de comprender que la didáctica se corresponde con el arte de enseñar de forma objetiva (Sarto, 2000). Se procura que los discentes aprendan diversos conocimientos base que les resultarán de utilidad durante toda su vida. No obstante, Sarto señala que esta disciplina no contempla la parte pasional y subjetiva, es decir, aquella que se relaciona con la voluntad y el interés por algo. Es en este último punto donde se sitúa la metodología de animación a la lectura 
(Sarto, 2000), a través de la cual se pretende ayudar a los educandos a cultivar su voluntad y a descubrir la lectura y el amor por ella.

En esta línea, se establecen objetivos básicos a conseguir:

1-. Acercar al alumno a la literatura mediante lecturas que pueda comprender. En cuanto a los textos, "la comprensión tiene que ver con los referentes tipográficos ([...] la estructura textual $[\ldots]$ ), el significado de las palabras (el vocabulario del lector, $[\ldots]$ los contextos semánticos, $[\ldots]$ ). Pero también afecta el modo de leerlos (realización de inferencias [...])" (Mata, 2008:130). En consecuencia, este conjunto de elementos determina si un texto puede llegar a comprenderse o no, pues si alguno de ellos es demasiado difícil de asumir, derivará en la incomprensión del texto. Por ello, a la hora de seleccionar un libro para una sesión de animación se deben tener en cuenta estos elementos, procurando que el libro no sea un reto insuperable para la comprensión del alumnado.

Sin embargo, existen otros factores disposicionales que influyen en la comprensión, como son: la motivación, el interés o el compromiso (Mata, 2008). Estos están ligados a la subjetividad mencionada anteriormente y es preciso cultivarlos para superar el esfuerzo que requiere la comprensión y poder descubrir el gusto por la lectura. En este sentido, las sesiones de animación contribuyen a profundizar en esa comprensión y ponen al servicio de los participantes espacios de evaluación formativa, donde el alumnado podrá ir recibiendo feedback para mejorar su comprensión.

2-. Procurar que los educandos disfruten. "[L]a palabra placer está irreduciblemente unida a la de lectura. El placer de leer [...] es una locución bien trabajada exitosa y tranquilizadora" (Mata, 2008:37). Sin embargo, esta expresión resulta engañosa a causa de las connotaciones del significado en relación con la palabra placer. Conviene aclarar que el placer de leer un texto "es siempre un placer radicalmente individual" (Mata, 2008:41). En otras palabras, con la animación a la lectura se persigue que los jóvenes descubran el placer individual que puede suponer la lectura de un texto. Sin embargo, en ocasiones se necesita un puente que permita a los lectores llegar a ese punto. Es ahí donde reside el aire de juego y diversión, que contienen las estrategias de animación.

$\mathrm{Al}$ contrario que la palabra placer, el término juego se relaciona con sentimientos positivos, duraderos y grupales. "El juego [...] es emoción, reto, posibilidades, opciones, imaginación, creatividad y conjunción de lo aprendido con lo nuevo" (González, Guízar, Sepúlveda y Villaseñor, 2003:53); además permite a los niños satisfacer sus necesidades y anhelos (Vygotski, 2000). Por consiguiente, el juego es una forma de conexión con sensaciones positivas y su adhesión a las estrategias de animación hace que esas emociones se relacionen con la lectura. No obstante, "el juego es un arma de dos filos, [...] pues si la animación se convierte en agitación el proceso se ha desvirtuado" (Sarto, 1984:23) y no cumplirá su función.

3-. Fomentar el proceso de reflexión del alumnado. Con esta finalidad, se debe favorecer que los estudiantes efectúen una lectura crítica, la cual permita al lector extraer datos e información, pero también "reflexiones complementarias que no son imprescindibles para la construcción del significado textual, pero que inevitablemente influyen en él" (Cassany, 2017:119). La lectura crítica y la realización de prácticas que incitan a la reflexión son imprescindibles para educar a los estudiantes, pues es necesario desarrollar la "capacidad de comprender diversas situaciones, tomar posturas, hacer elecciones conscientes, que nos ayuden a comprender y controlar la dirección que 
toma nuestra vida" (Serrano y Madrid, 2007:61).

Por otro lado, tras exponer los objetivos, cabe señalar que la ejecución de la sesión recae en una persona concreta que tiene que estar preparada y realizar su trabajo con entusiasmo. Este es el rol del animador, quien recibe dicho nombre porque se entiende que "la animación es un proceso de interiorización mediante el cultivo de la memoria, el entendimiento y la voluntad" (Sarto, 2004:106). Este debe conocer la literatura infantil en profundidad para poder ofertar al alumnado una selección de libros adecuados a sus características lectoras. Después de todo, se encarga de ayudar a los discentes a pasar de una lectura silenciosa e individual a un espacio agradable donde poner en común lo leído. En consecuencia, el animador debe conocer al grupo al que va a guiar, para así poder fijarse unos objetivos concretos a trabajar en cada una de las sesiones de animación, procurando el desarrollo de la autonomía personal del niño. La persona que asuma este rol debe tener una actitud alegre, abierta y creativa, pero también responsabilidad, orden, firmeza, sentido común, etc. (Sarto, 1984, 1998).

Finalmente, es preciso destacar la fundamentación del elemento clave de las sesiones de animación a la lectura, las estrategias. Estas son los procedimientos que ponemos en práctica para acercar a los jóvenes a la lectura. No obstante, la aplicación aislada de estrategias no producirá los efectos deseados; solo una aplicación constante puede conseguir que los discentes se afiancen en el proceso lector (Sarto, 1984). Asimismo, es importante que durante su implementación no se promueva la competición, sino la cooperación con el resto de compañeros y a la mejora personal.

\section{Propuesta didáctica}

La propuesta tiene como finalidad incentivar el gusto literario y se ha diseñado para un grupo concreto (véase epígrafe 2.2). Las estrategias de animación a la lectura empleadas en la misma se han extraído de la obra de Sarto (1998). No obstante, se han realizado una serie de adaptaciones con el fin de ajustarlas a las necesidades del grupo y procurar que la sesión fuera más enriquecedora y participativa. Por ello, se han seleccionado tres conjuntos de estrategias con distintos fines.

El objetivo de la primera actividad es rememorar las características de los personajes del libro y reflexionar en profundidad sobre ellas. Para ello, se ha realizado un compendio de las estrategias propuestas (Sarto, 1998). En concreto, se ha extraído la dinámica base de la estrategia número 54, la cual consiste en proporcionar al alumnado unas tarjetas que contengan fragmentos de los diálogos realizados por algunos personajes, de modo que a partir de su lectura, los discentes puedan determinar quién dice la frase y a quién se lo dice. Además, se incluye la fundamentación de las estrategias número nueve y 74 con el fin de tratar en profundidad todos los personajes. Estas trabajan las características descriptivas de los personajes y las cuestiones relativas a los sentimientos de estos, respectivamente.

A partir de estas ideas, se obtiene un ejercicio completo que permite: comprender lo leído; otorgar importancia a los sentimientos y actitudes que presentan los personajes; observar cómo dichos sentimientos y actitudes influyen en la forma de actuar de los personajes; favorecer la adquisición de valores; enriquecer la percepción del alumnado mediante la interacción con sus compañeros, y promover que el lector adquiera una conciencia crítica. 
En la segunda actividad, se han combinado las estrategias 12 y 35 (Sarto, 1998). El objetivo de aunar dichas estrategias radica en crear una actividad que permita ejercitar la comprensión lectora, cultivar la atención en la lectura realizada y valorar el orden en una narración. En este sentido, la primera de las estrategias mencionadas propone que los participantes ordenen distintos párrafos para formar un fragmento de la obra. Asimismo, la segunda estrategia insta al alumnado a indicar lo que sucede antes y después de un párrafo extraído de la obra a trabajar. En esta línea, para dinamizar el ejercicio se añade un elemento clave que permite la movilidad del alumnado y promueve la comunicación entre los estudiantes. Dicho elemento se basa en que la actividad sea realizada en un espacio muy delimitado. De este modo, los educandos, para conseguir ordenar el pasaje, necesitarán expresarse de forma oral con sus compañeros con la finalidad de: comunicar lo que ocurre en el fragmento de narración que tienen en su poder, expresar lo que creen que sucede antes y después para organizarlo, y consensuar un patrón de movimiento para poder llegar a desplazarse y conformar el texto.

Como actividad de cierre se ha seleccionado la estrategia número 41 (Sarto, 1998). Con esta actividad se tiene como finalidad realizar una evaluación general y formativa de la comprensión lectora. La estrategia consiste en que el alumnado recite adivinanzas sobre el libro seleccionado, por parejas, de forma que todos reciten y adivinen. En caso de que un estudiante no conozca la respuesta a su adivinanza, el compañero que la recitó debe ayudar a su pareja a través de la realización de mímica y sonidos. De esta forma se consigue: repasar contenidos clave de la obra que se hayan podido pasar por alto durante la sesión, asegurar que todos los discentes han comprendido la historia en su totalidad, desarrollar la lectura con entonación, favorecer la creatividad del alumnado, potenciar su imaginación y fomentar la colaboración entre compañeros.

Por último, como indica el método de Sarto (1984), se escoge una novela que se ajuste a la edad y al nivel de comprensión lectora de los estudiantes y sea idónea para desarrollar las estrategias seleccionadas. El libro elegido fue El hermano secreto de Caperucita Erre (Gómez, 2010). Esta elección también se vio motivada porque la obra es una versión renovada del cuento popular Caperucita Roja, el cual es muy conocido y próximo al público infantil. Además, a lo largo del desarrollo de la novela aparecen, como personajes, autores clave de la historia de la literatura (Charles Perrault y los hermanos Jacob y Wilhelm Grimm). La inclusión de estos personajes permite hacer partícipe al alumnado de una parte importante de la historia de la literatura.

\subsection{Contextualización}

La sesión de animación a la lectura se ha diseñado para el sexto curso de Educación Primaria de un centro educativo de carácter público situado en Segovia. Respecto a las características del centro, cabe añadir que consta de una única línea en todos los cursos y que actualmente el nivel sociocultural de las familias de los escolares es medio-alto.

El grupo al que va dirigida la sesión está formado por 22 estudiantes de 11 y 12 años. En cuanto al nivel de aprendizaje, se diferencian tres grupos: nivel alto, al que pertenece la mayoría de los discentes, quienes se caracterizan por su autonomía y progresión adecuada en la adquisición de la habilidad de comprensión lectora; nivel medio, formado por un pequeño grupo de estudian- 
tes, que tienen una actitud positiva y demuestran constancia a la hora de trabajar, pero presentan algunas dificultades para conseguir el nivel adecuado de comprensión lectora; y el nivel en proceso, el más reducido de todos los grupos, integrado por educandos a quienes les cuesta bastante trabajar y realizar tareas de comprensión lectora. Finalmente, el grupo participante tiene un nivel conductual correcto y positivo en el aula, así como una gran capacidad para trabajar en equipo.

\subsection{Puesta en práctica}

Esta experiencia se desarrolló desde el ámbito universitario, con el fin de dar a conocer la metodología de animación en el centro educativo al que está dirigida. Por este motivo, la sesión fue una actividad externa a la programación curricular del centro. Así pues, la experiencia se realizó en la biblioteca universitaria del Campus María Zambrano (Segovia). Para el desarrollo de la sesión se contó con la colaboración de dos alumnos del Grado de Educación Primaria que ejercieron de coordinadores y tres estudiantes más que fueron los animadores. La formación de coordinadores y monitores se realizó durante las clases de la asignatura de "Literatura Infantil" impartida en el Grado de Educación Primaria. Esta consistió en tres sesiones teóricas dedicadas al estudio del método de Sarto $(1984,1998)$ y la supervisión de un Proyecto de Aprendizaje Tutorado como evaluación de la adquisición de competencias.

Como paso previo al inicio de la ejecución de la animación, el centro universitario contactó con la escuela y se programó la sesión teniendo en cuenta las características del grupo. Seguidamente, se acordó la fecha de la sesión de animación, dando 15 días al alumnado para realizar la lectura silenciosa. El día previsto para el desarrollo de la sesión, los tres animadores, bajo la supervisión de los dos coordinadores, pusieron en práctica las tres actividades descritas. Para ello se dividió el grupo clase en tres subgrupos y a cada uno de ellos se le asignó un animador. De este modo, los tres subgrupos realizaron los tres ejercicios de forma simultánea. A continuación, se presenta el desarrollo de dichas actividades (Figuras 1-9).

\section{Actividad 1: compendio de estrategias sobre personajes. ¿Recuerdas a los personajes?}

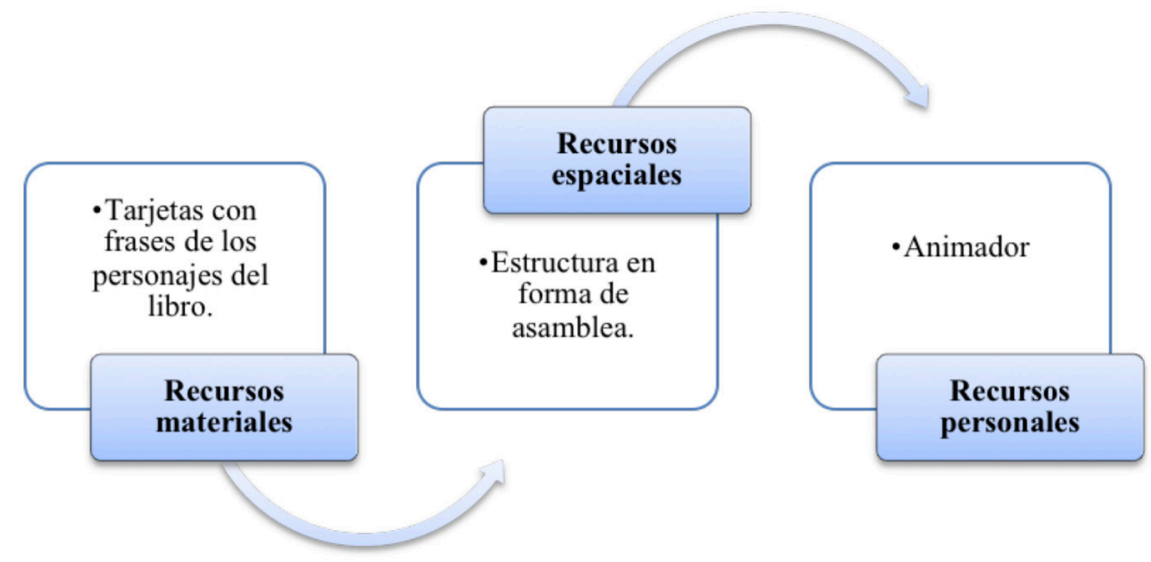

Figura 1. Recursos necesarios para la actividad, ¿recuerdas a los personajes? 
Pasos a seguir para desarrollar la actividad

- El animador repartirá una serie de tarjetas que contendrán diferentes frases extraídas del libro.

- Cada estudiante leerá su frase en silencio y con detenimiento.

- Tiempo de reflexión interna, con el fin de averiguar quién es el personaje que dice la frase y a quién va dirigida.

- Cada discente comparte su frase y reflexión con el grupo.

- Se crea un espacio de diálogo donde el mediador realiza preguntas oportunas para ayudar a los educandos a identificar los sentimientos y patrones psicológicos de los personajes.

Figura 2. Pasos a seguir para desarrollar la actividad, ¿recuerdas a los personajes?

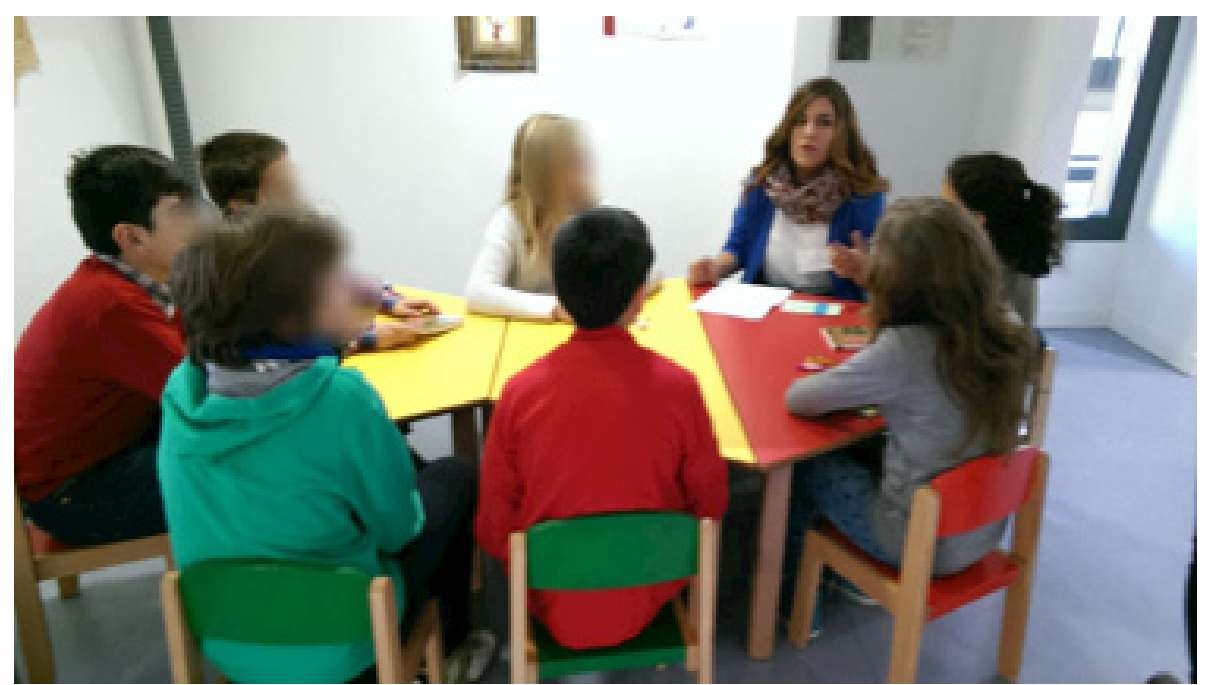

Figura 3. Grupo de estudiantes con una animadora.

Actividad 2: compendio de estrategias sobre el orden en una narración. Reconstruimos la historia.

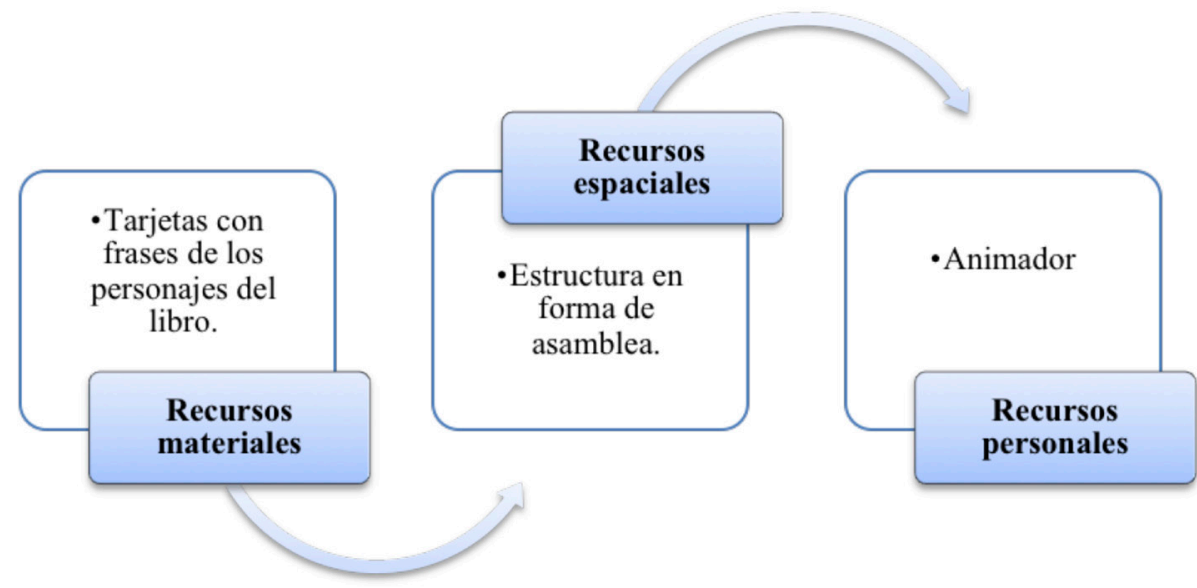

Figura 4. Recursos necesarios para la actividad, reconstruimos la historia. 


\section{Pasos a seguir para desarrollar la actividad}

- Los educandos se colocan sobre una circunferencia realizada en el suelo con cinta adhesiva.

- Se le proporcina a cada estudiante una tarjeta con un fragmento del libro.

- Los alumnos leen las tarjetas según el orden en el que se encuentran posicionados sobre la circunferencia.

- Se reflexiona sobre la alteración del orden de los fragmentos del libro.

- Se llega a un acuerdo sobre el orden correcto de los fragmentos que forman el pasaje.

-Se dialoga sobre posibles formas de desplazarse por la circunferencia para que cada estudiante alcance la posición correcta y el pasaje narrativo quede ordenado.

-Los educandos comienzan a desplazarse sin dejar de pisar la circunferencia.

-Se vuelven a leer todos los fragmentos para comprobar que el orden es correcto. Si fuera necesario se alteraría de nuevo el orden hasta llegar a la ordenación del pasaje.

Figura 5. Pasos a seguir para desarrollar la actividad, reconstruimos la historia. Fuente: propia a partir de Sarto (1990).

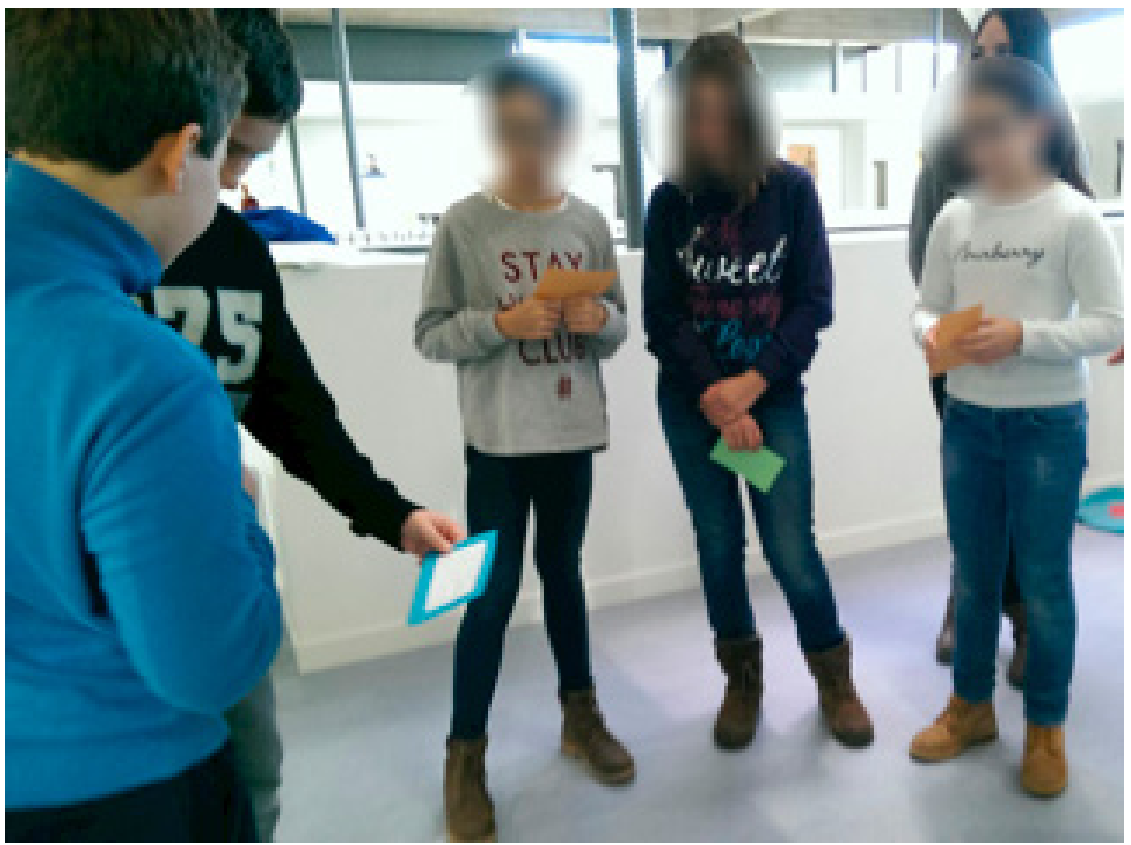

Figura 6. Grupo de estudiantes en la sesión de animación a la lectura. 


\section{Actividad 3: estrategia para evaluar la comprensión lectora. ¿Te lo sabes o te lo represento?}

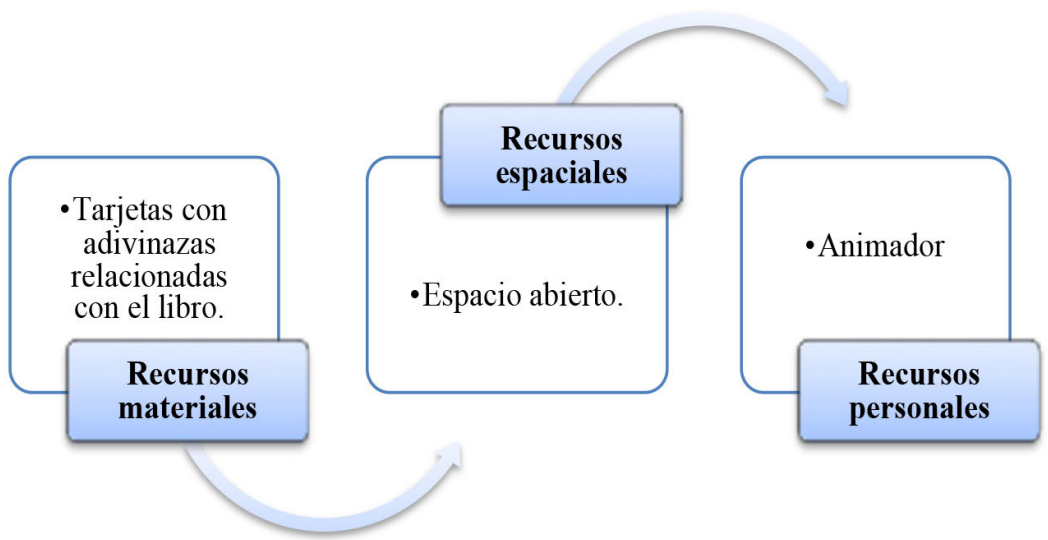

Figura 7. Recursos necesarios para la actividad, ¿te lo sabes o te lo represento?

Pasos a seguir para desarrollar la actividad

- Se divide al grupo-clase en parejas.

- Se entrega a cada discente una serie de tarjetas con adivinanzas sobre la lectura.

- Cada estudiante lee en voz alta las adivinanzas a su compañero, quien debe resolver la misma.

- En caso de que la respuesta no fuera correcta, el alumno que plantea la adivinanza tiene que representar la respuesta mediante mímica y sonidos. De esta forma ayuda a su compañero a refrescar su memoria hasta llegar a la respuesta correcta.

- Una vez resuelta la adivinanza, los estudiantes pueden comentar el fragmento del libro al que se refiere o solicitar apoyo para comprender mejor el mismo.

- Durante esta actividad los componentes de las parejas intercambiaran sus roles, de forma que ambos presenten y resuelvan las adivinanzas.

Figura 8. Pasos a seguir para desarrollar la actividad ¿te lo sabes o te lo represento?

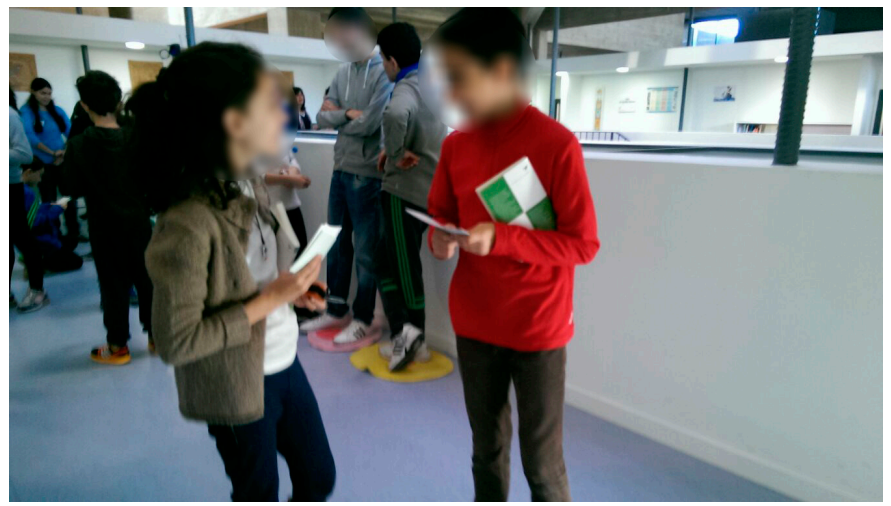

Figura 9. Dos escolares realizando la actividad, ¿te lo sabes o te lo represento? 


\section{Reflexión final}

Antes de concluir la sesión, conviene realizar una asamblea final para comprobar si se ha logrado el fin que se pretendía alcanzar con las estrategias. Con este objetivo, se pueden realizar al alumnado preguntas como: ¿habéis comprendido la lectura?, ¿habéis disfrutado leyendo el libro y participando en las actividades?, ¿os ha gustado reflexionar sobre la historia?, ¿os gustaría que estas actividades se realizaran en el colegio?, ¿os animaríais a leer otro libro para realizar otra sesión?...

\section{Resultados y análisis}

En base a la ORDEN EDU/519/2014, de 17 de junio, por la que se establece el currículo y se regula la implantación, evaluación y desarrollo de la educación primaria en la Comunidad de Castilla y León, y en concreto a lo regulado en su Anexo I-B acerca del bloque 5: Educación Literaria, del área de Lengua Castellana y Literatura, se puede afirmar que la animación a la lectura contribuye al desarrollo del gusto por la lectura y a la formación lectora a través del trabajo con obras literarias próximas a los educandos. En este sentido, la selección del libro El hermano secreto de Caperucita Erre fue acertada porque el alumnado lo consideró cercano a causa del paralelismo con el cuento popular Caperucita Roja. Esto es un proceso clave para evitar la desmotivación de los estudiantes que puede producirse "por los malos encuentros con los libros y las lecturas no deseadas" (Yubero y Larrañaga, 2010:16). Además, al no ser una actividad obligatoria los discentes han realizado la lectura con entusiasmo y han iniciado el camino hacia el gusto por la misma.

El modelo de estrategias de animación a la lectura empleado (Sarto, 1998) requiere que el alumnado tenga una experiencia individual y emotiva con el texto literario trabajado (Martín y Rascón, 2015). Como se ha comprobado en esta intervención puntual, el componente emocional es primordial porque establece una vinculación personal con la obra literaria y despierta el interés por la formación lectora (Sanjuán, 2013). Esta relación emocional con el libro trabajado, y su lectura individual, permitió lograr que los estudiantes realizaran un acercamiento personal a la lectura que resultó indispensable para la correcta ejecución de los ejercicios propuestos (Sarto, 1998).

Asimismo, se establece que la animación a la lectura "se apoya en estrategias educativas, creadas para desarrollar la capacidad lectora, en la puesta en común, en grupo, de un libro leído por todos individualmente, con la orientación del [...] mediador" (Sarto, 2004:105). En este sentido, durante esta experiencia puntual, se ha conseguido fomentar la puesta en común y el diálogo sobre los contenidos referentes al libro seleccionado. Este es un aspecto fundamental para lograr el objetivo de reflexionar (Sarto, 1998), pues es primordial destacar que "la literatura no venía de la autoridad del profesor o del currículo, sino de sentimientos humanos muy intensos. No era para ser individualmente estudiada sino colectivamente compartida" (Flecha, 1997:50).

Los estudiantes también tuvieron que comprender el contenido de los recursos y del libro para alcanzar correctamente las estrategias, logrando así otro de los objetivos planteados: acercar al alumno a la literatura mediante lecturas que pueda comprender (Sarto, 1998). Los discentes realizaron una buena comprensión inicial del libro, de modo que su selección fue adecuada al nivel. 
Asimismo, durante la puesta en común se profundizó en dicha comprensión a través de procesos de reflexión crítica, propiciando que los educandos mejoren sus habilidades de argumentación y razonamiento verbal.

Los resultados relacionados con el objetivo "disfrutar con la lectura", permiten afirmar que la propuesta de animación a la lectura diseñada ha logrado despertar el entusiasmo en los discentes gracias al trabajo del cuento escogido y del componente lúdico de las actividades efectuadas; esto ha permitido comprobar que el juego unido a la literatura ayuda a fomentar en "la población más joven el deseo de leer y el fascinante mundo que con la lectura de un libro, se despliega en su imaginación, como si de un juego se tratara" (Moreno y Valverde, 2004:169).

Por otro lado, se pasa a analizar cada una de las dinámicas diseñadas para esta sesión. La primera actividad, denominada “¿Recuerdas a los personajes?”, resultó sencilla para los educandos porque identificaron fácilmente tanto a los personajes, como sus respectivos sentimientos. Esto permite deducir que la lectura individual y silenciosa que ha realizado cada participante previamente, ha sido adecuada y determinante para esta dinámica.

No obstante, se apreció mayor dificultad en la identificación de algunos personajes secundarios y en el momento de rememorar algunos detalles puntuales y específicos de la obra. Esto podría deberse a que los educandos habían leído el libro con más de 15 días de antelación a la realización del taller, al contrario de lo que dictan las recomendaciones de los estudios de referencia (Sarto, 1998).Asimismo, durante la realización de este ejercicio, se hizo hincapié en que cada estudiante leyese su tarjeta en silencio y de manera reflexiva (Sarto, 1998) y procurase relacionar el diálogo con los personajes y escenas.

En la segunda actividad denominada "Reconstruimos la historia" se obtuvo un resultado menos satisfactorio, pues el ejercicio resultó más complejo. La dificultad encontrada en esta práctica radicó en la escasa habilidad del alumnado para mantener una gran capacidad de atención y retener la información que leía cada uno de los miembros del grupo; sin embargo, "las estrategias no son recetas" (Sarto, 1998:25). Por este motivo, se adaptó la actividad dedicándola más tiempo, para que el estudiantado se esforzase por lograr el objetivo de ordenar el pasaje. Esta modificación permitió que los discentes relacionasen los párrafos, después de realizar varias lecturas de los mismos, y los ordenasen progresivamente, ampliando el elemento motriz de la actividad durante gran parte del ejercicio.

La tercera estrategia llamada “¿Te lo sabes o te lo represento?”, favoreció el recuerdo de ideas e información de la obra literaria que aún no se habían trabajado de una forma lúdica y creativa. Al realizar estos ejercicios, se ha comprobado que las estrategias lúdicas empleadas en esta intervención puntual de animación a la lectura ayudan al lector

...a convertir la lectura en una experiencia significativa; a reconocer, contactar y expresar sentimientos y emociones; a la reflexión; a la conexión con sus vivencias personales y con otras previas, que en algunos casos inclusive lo llevarán a encontrar respuesta a cuestionamientos añejos y, por supuesto, al desarrollo de destrezas de pensamiento crítico y creativo. Así, el libro-objeto deja de serlo y se establece un juego de provocaciones mutuas de todo tipo que llevan al conocimiento. (González et al., 2003:56) 
Cabe añadir que las respuestas del alumnado permitieron comprobar cómo se produjo un acercamiento real entre estos y el texto literario, el cual ha contribuido al fomento del gusto por la literatura. Debido a esto, se puede afirmar que el objetivo propuesto por Sarto y por la Orden EDU 519/2014 se ha conseguido.

Respecto al papel de los animadores, se puede resaltar que las personas que dirigían los grupos intentaban fomentar la participación de todos los niños mediante breves intervenciones. Asimismo, los animadores consiguieron propiciar una conexión entre el estudiante y el libro, tratando de fomentar su capacidad cognitiva (Sarto, 1998).

\section{Conclusión}

Esta experiencia didáctica permite observar que los estudiantes se muestran entusiasmados con la lectura al emplear técnicas de animación, como el modelo de Sarto $(1984,1998)$ y separando estas de la rutina de clase y los trabajos obligatorios. En este caso, se han trabajado varias estrategias de dicha autora para mostrar a los estudiantes y docentes del centro educativo participante que las técnicas de animación a la lectura propician el acercamiento del alumnado a la lectura. En líneas generales, se puede deducir que este proceso capta la atención de los estudiantes, ya que durante la realización de estas actividades se mostraron entusiasmados, atentos y participativos, generando una predisposición positiva a continuar realizando sesiones de animación lectora.

La experiencia realizada ha demostrado que si los estudiantes comprenden las obras literarias son capaces de reflexionar sobre ellas y de disfrutar leyendo, logrando así despertar en ellos el interés por la lectura, lo cual es el inicio para generar el hábito lector. Por tanto, el resultado satisfactorio de la realización de esta experiencia puntual señala la validez del método empleado para avivar el gusto por la lectura (Sarto, 1998). Por consiguiente, la animación lectora, al poseer un aire lúdico, consigue despertar la curiosidad en los estudiantes y ayudarles a conocerse a sí mismos. Este aspecto es de vital importancia porque "si somos capaces de despertar esa curiosidad, el resto lo hará directamente el niño, [...] por el deseo de dar respuesta a sus inquietudes, a sus necesidades" (Beltrán y Téllez, 2002:10).

Estas conclusiones poseen ciertas limitaciones porque la muestra es muy reducida; por este motivo, la idoneidad de la propuesta de animación podría evaluarse en un futuro poniéndola en práctica en otros grupos y centros escolares, y mediante la selección de diferentes obras literarias. Incluso, la realización continuada de este tipo de experiencias a lo largo del tiempo podría permitirnos estudiar sus efectos en el hábito lector y en el gusto por la lectura de escolares de diferentes edades.

\section{Agradecimientos}

La autoría agradece su colaboración al centro participante y a la Dra. María Ángeles Martín, por su orientación y consejos. 


\section{Referencias bibliográficas}

Artola, T., Sastre, S., y Barraca, J. (2016). Diferencias de género en actitudes e intereses lectores: una investigación con alumnos españoles de primaria. Bordón. Revista de Pedagogía, 69(1), 11-26.

Avendaño, C. (2017). Motivación por la lectura: claves para entender su importancia a partir de una revisión conceptual. Revista Estudios Hemisféricos y Polares, 8(4), 1-19.

Becker, M., McElvany, N., y Kortenbruck, M. (2010). Intrinsic and Extrinsic Reading Motivation as Predictors of Reading Literacy: A Longitudinal Study. Journal of Educational Psychology, 102(4), 773-785. doi: 10.1037/a0020084

Beltrán, S., y Téllez, J. (2002). El papel de la escuela y la familia en la animación y el aprendizaje de la lectura. En D. del Río, B. Álvarez, S. G. Beltrán y J. A. Téllez: Orientación y Educación Familiar. Madrid: UNED. Recuperado de https://goo.gl/FKvqTm

Cassany, D. (2017). Aproximaciones a la lectura crítica: teoría, ejemplos y reflexiones. Tarbiya, Revista de investigación e innovación educativa, 32, 113-132. Recuperado de https:// revistas.uam.es/tarbiya/article/view/7275/7623

Flecha, R. (1997). Compartiendo palabras. El aprendizaje de las personas adultas a través del diálogo. Barcelona: Paidós.

Gambrell, L. B. (2011). Seven rules of engagement. What's Most Important to Know About Motivation to Read. The Reading Teacher, 65(3), 172-178. doi:10.1002/TRTR.01024

Gómez, R. (2010). El hermano secreto de Caperucita Erre. Zaragoza: Edelvives.

González, R., Guízar, M. Sepúlveda, I., y Villaseñor, L. (2003). La lectura: vinculación entre placer, juego y conocimiento. Sinéctica, 0(22), 52-57. Recuperado de https://sinectica.iteso.mx/ index.php/SINECTICA/article/view/316/309

Guthrie, J. T., y Wigfield, A. (2000). Engagement and motivation in reading. In M. L. Kamil, P. B. Mosenthal, P. D. Pearson y R. Barr (Eds.), Reading research handbook (Vol. 3, pp. 403422). Mahwah, NJ: Erlbaum.

Gutiérrez, R. (2016). La lectura dialógica como medio para la mejora de la comprensión lectora. Investigaciones sobre lectura, 5, 52-58. Recuperado de http://rua.ua.es/dspace/ handle/10045/62508

Mata, J. (2008). 10 ideas clave. Animación a la lectura. Hacer de la lectura una práctica feliz, transcendente y deseable. Barcelona: Graó.

Martín, M. A., y Rascón, D. (2015). La educación literaria: una oportunidad de aprendizaje servicio para la formación integral del futuro maestro. Revista de Currículum y Formación del Profesorado, 1(19), 350-366.

McGeown, S. P., Duncan, L. G., Griffiths, Y. M., y Stothard S. E. (2015). Exploring the relationship between adolescent's reading skills, reading motivation and reading habits. Reading \& Writing, 28(4), 545-569. Doi:https://doi.org/10.1007/s11145-014-9537-9

McGeown, S. P., Norgate, N., y Warhurst, A. (2012). Exploring intrinsic and extrinsic reading motivation among very good and very poor readers. Educational Research, 54(3), 309-322. Doi: https://doi.org/10.1080/00131881.2012.710089

Moreno, C., y Valverde, R. (2004). Los cuentos y juegos, carácter lúdico necesario como recurso didáctico para la animación a la lectura. Glosas didácticas, 11, 169-176. Recuperado de http://www.um.es/glosasdidacticas/doc-es/14Carmelo.pdf

OCDE. (2015). PISA 2015. Resultados clave. OCDE. 
ORDEN EDU/519/2014, de 17 de junio, por el que se establece el currículo y se regula la implantación, evaluación y desarrollo de la educación primaria en la comunidad de Castilla y León, 4181-44625.

Sanjuán, M. (2013). La dimensión emocional en la educación literaria. Zaragoza: Prensas de la Universidad de Zaragoza.

Sarto, M. (1984). La animación a la lectura para hacer al niño lector. Madrid: SM.

Sarto, M. (1998). Animación a la lectura con nuevas estrategias. Madrid: SM.

Sarto, M. (2000). De dónde viene y a dónde va la animación a la lectura. Educación y futuro: revista de investigación aplicada y experiencias educativas, 2, 45-51. Recuperado de https:// dialnet.unirioja.es/servlet/articulo?codigo $=2043920$

Sarto, M. (2004). Hacia otra forma de leer. Educación y Biblioteca, 140, 104-106. Recuperado de https:/gredos.usal.es/jspui/bitstream/10366/119060/1/EB16 N140 P104-106.pdf

Serrano, S., y Madrid, A. (2007). Competencias de lectura crítica. Una propuesta para la reflexión. Acción pedagógica, 16, 58-68. Recuperado de https://www.saber.ula.ve/ bitstream/123456789/17304/2/articulo6.pdf

Vygostki, L. S. (2000). El desarrollo de los procesos psicológicos superiores. Barcelona: Crítica.

Wigfield, A., y Eccles, J. S. (2000). Expectancy-Value Theory of Achievement Motivation. Contemp Educ Psychol, 25(1), 68-81. Doi: 10.1006/ceps.1999.1015

Yubero, S., y Larrañaga, E. (2010). El valor de la lectura en relación con el comportamiento lector. Un estudio sobre los hábitos lectores y el estilo de vida de los niños. Revista OCNOS, 6, 7-20. Recuperado de https://www.revista.uclm.es/index.php/ocnos/article/download/ ocnos 2010.06.01/168 